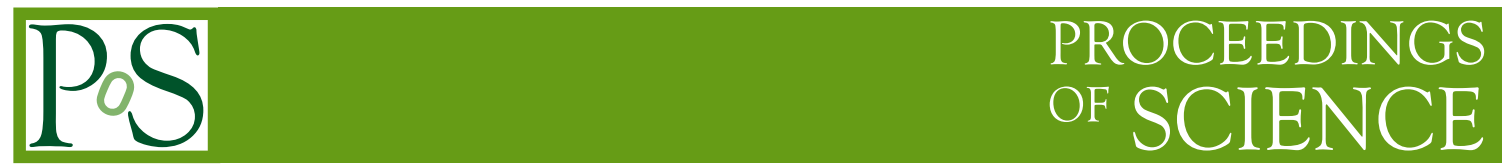

\title{
Galactic and Extragalactic Novae - A Review
}

\section{Rosa Poggiani*}

Università di Pisa and Istituto Nazionale di Fisica Nucleare, Sezione di Pisa

E-mail: rosa.poggiani@unipi.it

\begin{abstract}
This paper is a short and updated version of the review [101] about the observations of novae over the electromagnetic spectrum. The review will briefly discuss the new findings in the photometric and spectroscopic evolution of galactic and extragalactic novae, their high energy emission, their distances, in the context of present ground and space based instruments.
\end{abstract}

Accretion Processes in Cosmic Sources - II - APCS2018

3-8 September 2018

Saint Petersburg, Russian Federation

${ }^{*}$ Speaker. 


\section{Introduction}

Classical novae are cataclysmic variable systems where a white dwarf is accreting material from a secondary star [86], [14], [9]. Novae show outbursts with a fast increase of optical brightness, produced by a ThermoNuclear Runaway (TNR) on the surface of the white dwarf [14], [9], [137], [39]. The historical observations of nova outbursts were performed in the optical domain, that is presently explored with high cadence photometry and spectroscopy. The emission of novae extends over the whole electromagnetic spectrum, making them multifrequency sources [41]. The availability of X-ray and gamma ray observatories has allowed the investigation of several physical processes. Novae have also been discussed as possible distance indicator. An introduction to the problems related to cataclysmic variables and novae can be found in [41].

In the present paper, I review some aspects of multifrequency observations of Galactic and extragalactic novae, discussing the recent findings of high cadence optical observations and high energy X-ray and gamma ray instruments.

\section{Novae: optical properties}

Catalogs of Galactic novae have been built by different authors [37], [36], [103], [104], [74]. To date, more than four hundreds Galactic novae are known. The majority of extragalactic novae has been observed in the close galaxies [87], among them more than one thousand novae in M31 ${ }^{1}$, about fifty in $\mathrm{M}_{3}{ }^{2}$ and $\mathrm{LMC}^{3}$, about twenty in SMC ${ }^{4}$.

A distinctive feature of novae is a decline of the light curve after the outburst. The decline is not necessarily smooth, but can show oscillations, dips, flares etc. The photometric evolution of novae has been discussed by [138], who classified them into seven prototype classes (Fig. 1): the F-class novae with flat top ( $2 \%$ of all novae); the C-class with a cusp (1\%); the J-class with jitters $(16 \%)$; the P-class with a plateau (21\%); the O-class with oscillations (4\%); the S-class with a featureless decline (38\%); the D-class with dips (18\%). The decline of S-class novae in time is described by an universal law in the model by [44], [58].

The archives of AAVSO ${ }^{5}$ and VSNET ${ }^{6}$ report photometric observations of a large number of novae. The new all-sky surveys operating with high temporal cadence are providing new insights about the physical properties of novae, among them the orbital period and the pre-maximum stage. The Solar Mass Ejection Imager (SMEI), with a time cadence of about 100 minutes, has measured the light curves of 13 galactic novae [50], [51], including a few systems whose maximum had been missed by ground based instruments. Some novae have been observed during the poorly studied pre-maximum stage (see [100] for a review of the pre-maximum stage). The ground based Optical Gravitational Lensing Experiment (OGLE) has observed 39 Galactic novae [71] (about 30\% of them original discoveries by the survey), 80 post-nova candidate systems and estimated the orbital periods of 18 objects.

\footnotetext{
${ }^{1}$ http://www.mpe.mpg.de/ m31novae/opt/m31/index.php

${ }^{2}$ http://www.mpe.mpg.de/ m31 novae/opt/m33/index.php

${ }^{3}$ http://www.mpe.mpg.de/ $\sim$ m31 novae/opt/lmc/index.php

${ }^{4}$ http://www.mpe.mpg.de/ m31 novae/opt/smc/index.php

${ }^{5} \mathrm{https}$ ://www.aavso.org/

${ }^{6}$ http://ooruri.kusastro.kyoto-u.ac.jp/mailman/listinfo/
} 


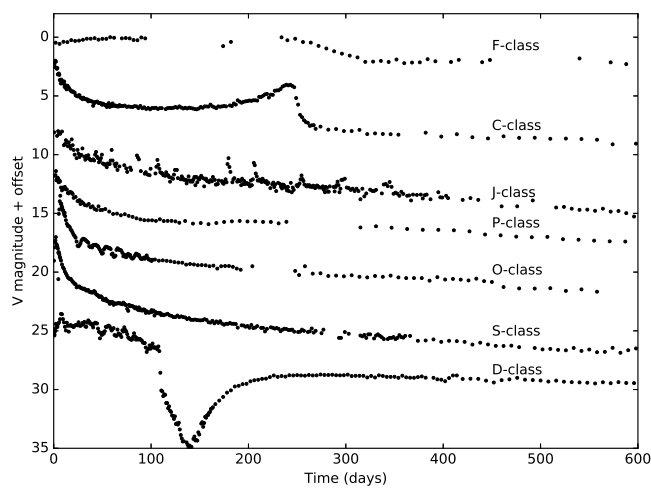

Figure 1: Classification of the light curves of novae, after [138]; data from [138]

Novae have been traditionally divided into different spectral classes. The original spectral classification by [150], [151], [152], based on the presence of specific non-Balmer lines during the early post-outburst stage, includes two classes, Fe II novae and He/N novae, that show Fe II multiplets or He and $\mathrm{N}$ lines, respectively. Fe II novae account for about $80 \%$ of Galactic novae, are governed by wind ejection and evolve towards late spectra with nebular lines. He/N novae are governed by shell ejection and evolve towards nebular spectra with coronal lines. An example of the post-outburst spectra of Fe II and He/N novae is shown in Fig. 2. Fe II and He/N novae re different also from the point of view of photometric evolution: the latter are faster and brighter than the former [34] and concentrated close to the Galactic plane [28], [34].
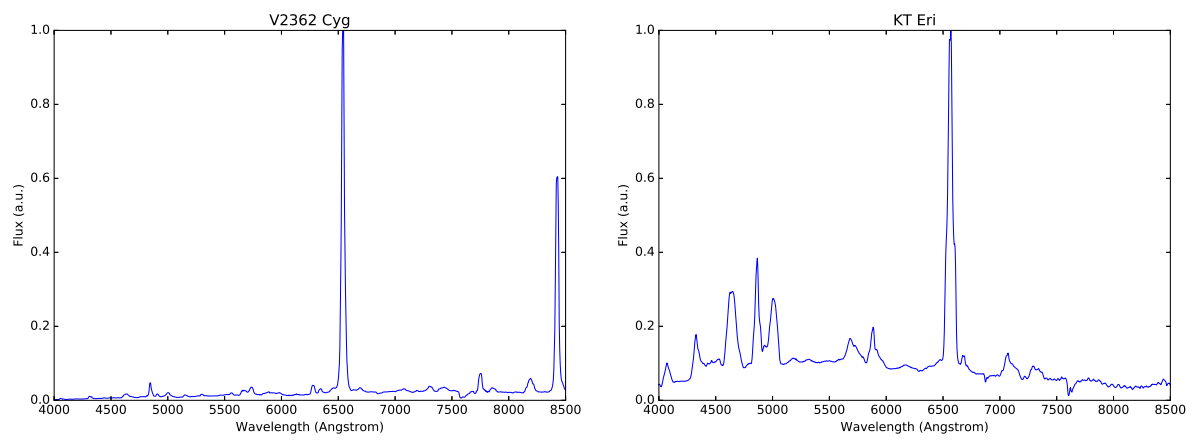

Figure 2: Spectra of the Fe II nova V2362 Cyg [91] and of the He/N nova KT Eri (Poggiani, in preparation) during the initial decline

Some novae since show spectra evolving from the Fe II to He/N class, defining the class of hybrid novae. V5558 Sgr [140] and T Pyx (see e.g. [54], [40]) switched from a He/N spectrum to a Fe II spectrum. According to [155], the origin of the two classes of spectra is in the ejecta of the white dwarf or in a circumbinary gas envelope around the companion. The two types of spectra can appear at different times according to the relative contribution of the above processes [155].

The investigation of the physical properties of novae requires catalogs of spectra secured at different times during the evolution. The SMARTS Consortium [148], [149] is building a spectral atlas of Southern novae, on the path of the Tololo nova atlas [152], [153], using the ANDICAM dual channel imager and the RC spectrograph at the SMARTS $1.5 \mathrm{~m}$ telescope. The atlas, that 
so far includes about one hundred novae, has been used for synoptic investigations of the relation between the $\mathrm{K}_{s}$ band flux and dust formation and the link between He II 4686 in He/N novae and the super-soft phase in the X-rays [149].

Since 2005 I am monitoring Northern novae at the Cassini 1.5m telescope, equipped with the BFOSC Imager/Spectrograph [97]. Optical spectra are secured during the decline with a high cadence. The sample includes more than twenty galactic novae and a few extragalactic novae: V1663 Aql [88], V1722 Aql, V809 Cep, V962 Cep, V2362 Cyg [91], V2467 Cyg [92], V2468 Cyg, V2491 Cyg, V2659 Cyg, V407 Cyg, V339 Del, KT Eri, V959 Mon, V2615 Oph, V2670 Oph [93], V2944 Oph, V496 Sct, V612 Sct (ASASSN-17hx) [100], V556 Ser, V5558 Sgr [89], [94], V5584 Sgr [96], [99], V458 Vul [90], V459 Vul [95], the extragalactic novae M31 2009-10b, M31 2010-07a, M31 2011-07 and M33 2010-07a [98]. V458 Vul is an hybrid nova that switched from the Fe II to the He/N class after the outburst [90]. V2362 Cyg showed a cusp explained by a secondary mass ejection [91]. V2467 Cyg showed oscillations during the initial decline [92]. V5558 Sgr is a very slow nova with a white dwarf with a mass at the limit to trigger a nova outburst [89], [94]. V612 Sct (ASASSN-17hx) is a slow nova with a long lasting pre-maximum stage [100]. M31 2009-10b is one of the most luminous novae ever observed in M31 [98], while M33 2010-07a is the first nova in M33 showing a secondary mass ejection [98].

According to the number of observed eruptions, some objects are classified as recurrent novae [9], with recurrence times of outbursts of the order of decades. To date, there are 10 known recurrent Galactic novae [110], 4 in LMC [80] and 12 in M31 [122]. T Pyx, IM Nor and CI Aq1 have orbital periods smaller than one day and main sequence secondary stars; U Sco, V394 CrA and V2487 Oph have orbital periods of the order of one day and evolved secondaries; T CrB, RS Oph, V745 Sco and V3890 Sgr have orbital periods of the order of years and red giant secondaries. Most recurrent novae belong to the He/N spectral class. The recurrence time is determined by the white dwarf mass and the accretion rate [156]. Recurrent novae have an high rate of mass transfer explaining the short recurrence times [80] and are candidate progenitors of type Ia supernovae [139], [122]. The photometric history of Galactic recurrent novae has been presented by [110].

The fraction of recurrent novae in M31 is of the order of a few percent [122]. Nova M31 200812a, a supernova Ia progenitor candidate, underwent several recorded outbursts, due to the short recurrence time, of the order of one year [48]. The multifrequency observations of the outbursts with ground and space based instruments are reported by [25], [55], [10].

\section{Galactic and extragalactic nova rates}

The properties of extragalactic novae have been reviewed by [121]. The surveys of the close galaxies has produced nova rates for M31, M33, LMC, M87. In the following, an update of the nova rates in some close galaxies will be given. The existence of two distinct nova populations in M31 (disk and bulge) has been proposed by [24]. The spectroscopic survey of the M31 bulge by [143] was consistent with the spectral evolution of Galactic novae. The survey by [118] showed that the fraction of $\mathrm{Fe}$ II and $\mathrm{He} / \mathrm{N}$ novae were $82 \%$ and $18 \%$, as for Galactic novae. The survey of novae in M33 by [119] showed that 5 and 2 novae out of 8 were He/N and Fe II novae, respectively, in disagreement with the Galaxy and in M31 populations. The survey of LMC novae by [120] found a comparable fraction of novae of $\mathrm{Fe}$ II and He/N novae. The large fraction of He/N novae in M33 
and LMC is explained by their young stellar population, leading to more massive white dwarfs and an higher number of recurrent novae.

The Galactic nova rate has been discussed by [123], who suggested a rate of $50_{-23}^{+31} \mathrm{yr}^{-1}$. A compilation of the Galactic nova rate estimations is reported in Table 1.

\begin{tabular}{lr}
\hline Authors & Rate $\left(\mathrm{yr}^{-1}\right)$ \\
\hline Allen 1954 [5] & 100 \\
Sharov 1972 [133] & 260 \\
Liller and Mayer 1987 [63] & $73 \pm 24$ \\
Della Valle 1988 [27] & $15 \pm 5$ \\
Ciardullo et al. 1990 [18] & 11 to 46 \\
van den Bergh 1991 [146] & 16 \\
Della Valle and Livio 1994 [32] & 20 \\
Hatano et al. 1997 [46] & $41 \pm 20$ \\
Shafter 1997 [115] & $35 \pm 11$ \\
Shafter 2002 [117] & $36 \pm 13$ \\
Matteucci et al. 2003 [66] & 25 \\
Mroz et al. 2015 [71] & $13.8 \pm 2.6$ \\
Shafter 2017 [123] & $50_{-23}^{+31}$ \\
\hline
\end{tabular}

Table 1: Galactic nova rates

The estimated nova rates in M31 are shown in Table 2. The saturation of photographic plates used in the early observations prevented the detection of novae in the nucleus in the surveys by [52], [6], [106], [107], [108]. Novae can indeed appear in any part of M31 [18], with a larger density in the halo and in the bulge [17], [12].

\begin{tabular}{lr}
\hline Authors & Rate $\left(\mathrm{yr}^{-1}\right)$ \\
\hline Hubble 1929 [52] & 30 \\
Arp 1956 [6] & 26 \\
Capaccioli et al. 1989 [12] & $29 \pm 4$ \\
Shafter and Irby 2001 [124] & $37_{-8}^{+12}$ \\
Darnley et al. 2006 [24] & $65_{-15}^{+16}$ \\
\hline
\end{tabular}

Table 2: Nova rates in M31

The nova rate in M33 should be higher than in M31, since it is an early type galaxy [29]. The estimated rates range from less than $0.4 \mathrm{yr}^{-1}$ [134] to $4.7 \pm 1.5 \mathrm{yr}^{-1}$ [29]. The most recent estimation by [154] is $2.5_{-0.7}^{+1.0} \mathrm{yr}^{-1}$. The estimations of the nova rates in LMC are of the order of a few novae per year: 2 to 3 novae per year [42], $2 \pm 1 \mathrm{yr}^{-1}$ [13], 2.5 $\pm 0.5 \mathrm{yr}^{-1}$ [30], 2.4 $\pm 0.8 \mathrm{yr}^{-1}$ [72]. The estimations of the rate in M87 are: $91 \pm 34 \mathrm{yr}^{-1}$ [116]; $154_{-19}^{+23} \mathrm{yr}^{-1}$ [23]; 363 ${ }_{-45}^{+33} \mathrm{yr}^{-1}$ [128].

The estimated nova rates are compared the using Luminosity Specific Nova Rates (LSNR), normalizing them to the surveyed stellar mass [121] through the galaxy K-band luminosity. The rates in galaxies belonging to different Hubble types has been discussed by [157], [66]. The nova rate is proportional to the K-band luminosity of the galaxy (Fig. 3). 


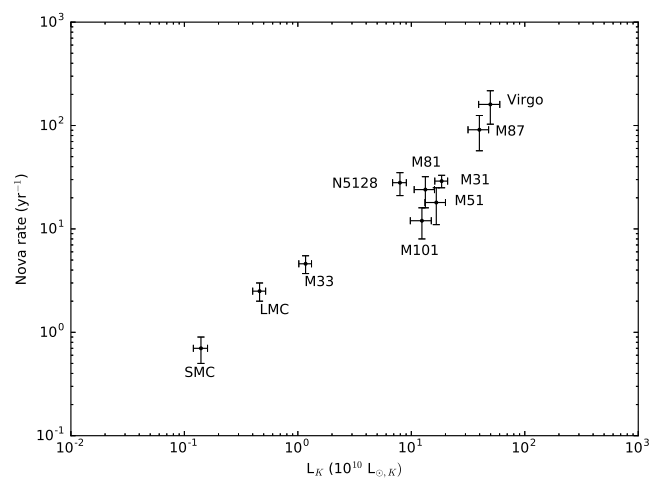

Figure 3: Relation between the nova rate in a galaxy and the K-band luminosity; data from [66]

\section{Novae and cataclysmic variables}

The orbital period has been estimated only for a small part of known novae. The distribution of measured periods, based on the data by [103], [104] is shown in Fig. 4, where novae with evolved secondary stars and periods above 10 hours have been excluded. The distribution is peaked in the region between 3 and 4 hours, above the period gap of cataclysmic variables. As discussed by [142], novae show an high mass accretion rate onto the white dwarf.

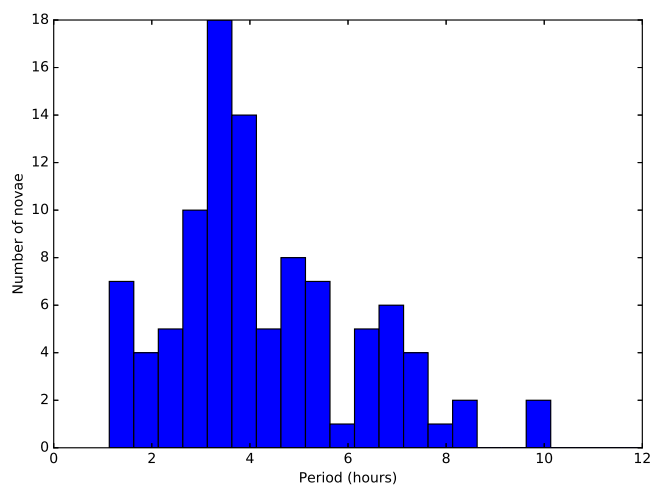

Figure 4: Distribution of the orbital periods of Galactic novae; data from [104]

The evolution of novae over long time scales has been discussed by [85]. The pre-outburst light curves discussed by [105] and [22] show that the majority of novae shares the same quiescent magnitude before and after the outburst [22]. It has been proposed that novae could hibernate in a low mass transfer state [125] after the high mass loss outburst event. The combined effect of the gravitational wave emission and magnetic braking during the hibernation stage progressively shrinks the orbital radius, reversing the detachment of the secondary star from its Roche lobe. The irradiation by the white dwarf produces a relatively high brightness for decades after the eruption before fading and varying the accretion rate, with the appearance of dwarf nova outbursts [125]. In the hibernation model novae show a cyclical evolution between high and low mass transfer states [125], with hibernation intervals larger than hundreds thousands years. The hibernation model predictions are in agreement with the observed decline rates of old novae [38] and the observation 
of shells around the dwarf novae Z Cam [126], AT Cnc [127] (whose last nova eruption occurred about three hundreds years ago [131]), the dwarf nova in Te 11 (associated to the nova $483 \mathrm{CE}$ in Orion) [69], the nova-like systems V1315 Aq1 [109] and IPHASX J210204.7+471015 [43]. V1213 Cen showed an awakening from the hibernation stage [73], showing dwarf nova outbursts with an amplitude of about three magnitudes before eruption. The nova that erupted on 11 March AD 1437 showed dwarf nova outbursts after five centuries [129].

The recover of old novae in the catalogs of cataclysmic variables [36] is difficult, since often the spectroscopic identification is missing. The mining of the plate archives of world observatories, whose records cover decades of observations, is being performed by [141], [53], [84]. The search for old novae is based on multicolour photometry followed by spectroscopic observations [141], [84]. Old novae are selected by their position in the color-color diagram, far from the main sequence curve [141]. The spectroscopic follow-up allows the classification of the object as a nova or a generic cataclysmic variable.

\section{Novae as distance indicators}

Novae have been considered in the past as candidate standard candles for the estimation of extragalactic distances [144], [33]. The Very Large Telescope (VLT) has been used to search for novae in NGC 1316, an early type galaxy in the Fornax cluster [31] at about $20 \mathrm{Mpc}$.

The method of the Maximum Magnitude Rate of Decline (MMRD) [21], [33], [26], [35], [112] has been recently criticized. A new class of faint and fast novae has been discovered in the high temporal cadence survey of M31, M81, M82, NGC 2403, NGC 891 [57]. The luminosity and decay time of the novae were not consistent with the standard MMRD relation. The detected fast and faint novae were Fe II novae, not He/N as in the Galaxy. Faint and fast novae are about $40 \%$ of novae in M87, with a large scatter around the MMRD relation [130]. The faint and fast novae could be associated to hot and massive white dwarfs [57], [156]. The nova outburst is dominated by the white dwarf mass [64], but the role of composition, temperature and accretion rate must be included [57]. The identity of the absolute magnitude achieved 15 days after the maximum by all novae [11], [12], [20], [35], [145] is supported by the observation of M87 novae [132].

A new distance estimation method has been presented by [83], who reported the distance of 73 novae. The approach relies on the location of the red clump giants in the infrared color-magnitude diagram and on the combination of reddening-distance relations with independent reddening estimates. The method has been checked against the distance of novae with measured expansion parallax [35]. The first data release of Gaia has produced parallax estimations for 16 cataclysmic variables [102], among them three old novae. The Gaia DR2 release has been used to estimate the distance of 18 old novae [114] and 41 novae [111].

\section{X-ray and gamma ray observations}

The availability of X-ray and gamma ray observatories has allowed the investigation of shocks in the nova processes. Due to the presence of accreting white dwarfs, novae are X-ray emitters. The first X-ray observations with EXOSAT [77] and ROSAT [59] showed flux variations during the nova outburst and a spectral hardness evolving towards a super-soft phase. The hard and soft 
components are associated to shocks within the ejecta and to the nuclear burning on the white dwarf surface. Swift has produced high cadence light curves of novae, starting a few days after the outburst, for a large number of novae in the Galaxy and in the close galaxies M31, LMC, SMC [82]. The high spectral resolution gratings of XMM-Newton and Chandra instruments [79], [75] has allowed the discovery of a large number of emission lines. As an example, the X-ray light curve of RS Oph [113] is reported in Fig. 5.

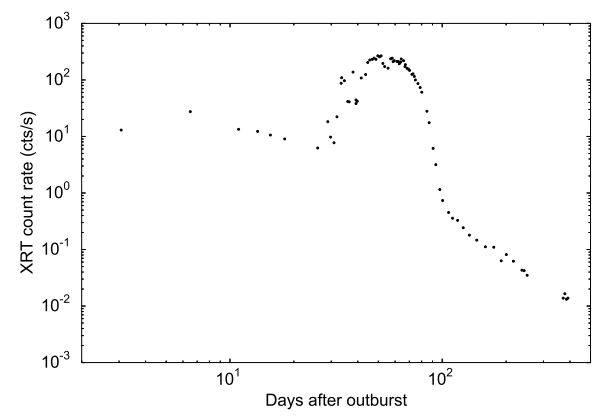

Figure 5: The Swift light curve of RS Oph in the 0.3-10 keV band; data from [113]

The 2006 outburst of RS Oph showed the occurrence of shocks in the ejecta [7], [136], [81]. The radio interferometry [76] and HST observations [8] suggested that the shock showed a bipolar geometry with an equatorial ring. After the nova eruption, the keV X-ray flux initially declined, before peaking at about day 30, the beginning of the super-soft phase. The shrinking of the photosphere and the decrease of the optical depth exposed the hotter layers. The burning ended around day 80 and caused the fading of the X-ray flux.

Swift has detected the super-soft phase, often associated with the optical forbidden [Fe X] 6374 line $\AA$, in several novae [113]. Galactic and M31 novae with a fast decline in the optical domain show high ejection velocities and an early turn-on and turn-off of the super-soft phase [47]. The high resolution X-ray spectra show the continuum and the white dwarf absorptions or broad emission lines or a combination of the above features [79]. The first observations of the thermal bremsstrahlung continuum now are replaced by the large number of lines observed in the present high resolution instruments [78]. Collisional plasmas explain the spectra outside the super-soft phase [75]. While shocks are responsible for the emission component before the beginning of the super-soft stage, the later emission is produced by the radiatively cooling thin ejected material [75]. The turn on and off times of the super-soft phase have been modeled by [44], [58] [45].

The detection of gamma rays from novae was expected in the $\mathrm{MeV}$ region, in association with positron annihilation and nuclear de-excitation in nitrogen, oxygen and sodium [19], [49]. The first unexpected detection of gamma rays in a nova occurred at higher energies, when the FermiLAT instrument observed the radiation of the symbiotic nova V407 Cyg in the region from 0.1 to $10 \mathrm{GeV}$ [1]. The high energy emission is explained by the interaction of material in the nova shell with the environmental medium of the red giant secondary. V407 Cyg is a symbiotic nova, where ejecta expand in a dense circumstellar wind and particles acceleration can occur in a blast wave. In addition to V407 Cyg, the Fermi-LAT detected GeV gamma ray emission in the classical novae V959 Mon, V1324 Sco and V339 Del [2], V1369 Cen and V5668 Sgr [15], V407 Lup [16], 
ASASSN-16ma [60], ASASSN-17mt [62], ASASSN-18fv [56]. As an example, the GeV light curve of V959 Mon is shown in Fig. 6.

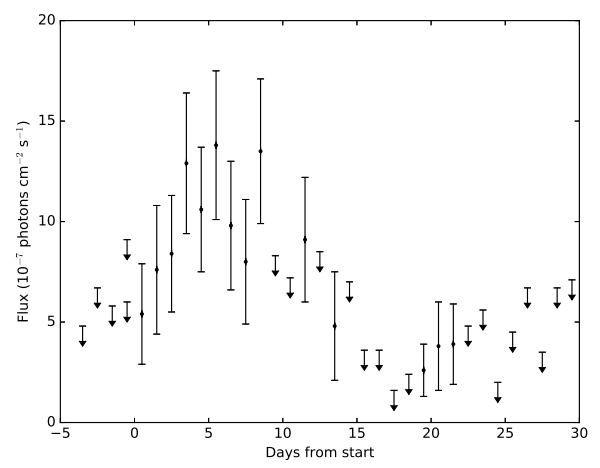

Figure 6: Fermi-LAT light curve (>100 MeV) [2]; data from [2]

Since the environmental density around classical novae is smaller than for symbiotics, the acceleration is produced by a bow shock of the ejected material in the ISM or by turbulence related small shocks [2]. Gamma ray emission has been discussed by the hadronic and the leptonic models [2]. In the former model, nova ejecta interact with nuclei in the environmental medium (novae) or with the stellar wind (symbiotics), producing neutral pions decaying into photons. In the latter model, gamma rays are produced by the interaction of accelerated electrons with photons through inverse Compton or with atoms through bremsstrahlung.

The observation of $\mathrm{GeV}$ gamma ray emission in different novae and symbiotic novae suggests that all novae could potentially be gamma ray emitters. Excluding the symbiotic nova V407 Cyg, the Fermi-LAT instrument detected gamma rays from six novae, out of the 69 novae discovered during the same interval [70]. According to [70], novae with a magnitude $\mathrm{R} \leq 12$ and within $8 \mathrm{kpc}$ are potentially detectable by the Fermi-LAT instrument.

Novae with gamma ray emission are suitable systems to investigate relativistic particle acceleration at shocks [67]. The high density of ejected material at the epoch of gamma ray emission produces radiative shocks. The fraction of the shock power spent in the acceleration of relativistic particle is governed by the ratio of gamma ray and optical luminosity, that was larger than the value estimated from cosmic ray observations in V1324 Sco and V339 Del. The model predicts X-ray radiation in the range from 10 to $100 \mathrm{keV}$, potentially detectable by NuSTAR, in coincidence with $\mathrm{GeV}$ emission [67]. The model for hard X-ray and gamma ray emission by [147] suggests that only a limited fraction of the gamma ray luminosity falls in the NuSTAR energy band, but with the clear signature of a zero spectral slope. The GeV emission of novae and the link to particle acceleration at shocks has been addressed by [65], who suggested that it is mainly produced from particles accelerated up to $100 \mathrm{GeV}$ at the reverse shock and linked to hadronic interactions in the cooling layer downstream of the shock. The gamma rays from ASASSN-16ma correlated with optical emission, suggesting the role of reprocessed emission from shocks [61] and supporting hadronic models for the gamma ray emission. The shocks and the relativistic particle acceleration in the nova eruption can potentially produce gamma ray emission in the $\mathrm{TeV}$ region, with a maximum gamma ray energy in the interval from $10 \mathrm{GeV}$ to $100 \mathrm{TeV}$ [68], the region of ground based atmo- 
spheric Cherenkov detectors, such as MAGIC or the future Cherenkov Telescope Array CTA [3]. The Cherenkov instrument MAGIC has monitored V339 Del in the energy region above $50 \mathrm{GeV}$, without significant detections [4]. The $\mathrm{TeV}$ gamma rays could be associated with $\mathrm{TeV}$ neutrinos, potentially detectable with IceCube [135].

\section{Conclusions}

Novae are multifrequency laboratories for accretion processes that are governed by a great number of astrophysical processes. The recent observations with high temporal cadence and at high energies are providing new insights into the outburst and the successive evolution.

\section{Acknowledgments}

The author thanks the organizers for the invitation to the conference. Thanks to Ivan Bruni for the assistance and to the Time Allocation Committee of the Loiano telescope for the observing time.

\section{References}

[1] A. A. Abdo et al., Sci 329 (2010) 817.

[2] M. Ackermann et al., Sci 345 (2014) 554.

[3] M. Actis et al., ExA 32 (2001) 143.

[4] M. L. Ahnen et al., $A \& A \mathbf{5 8 2}$ (2015) 582.

[5] C. W. Allen, MNRAS 114 (1954) 387.

[6] H. C. Arp et al., AJ 61 (1956) 15.

[7] M. F. Bode et al., ApJ 652 (2006) 629.

[8] M. F. Bode at al., ApJ 665 (2007) L63.

[9] M. F. Bode and A. Evans, Classical Novae, Cambridge University Press (2012).

[10] D. Boyd et al., ATel 11116 (2017).

[11] W. Buscombe and G. de Vaucouleurs, Obs 75 (1955) 170.

[12] M. Capaccioli et al., AJ 97 (1989) 1622.

[13] M. Capaccioli et al., ApJ 360 (1990) 63.

[14] A. Cassatella and R. Viotti, Physics of Classical Novae, Springer-Verlag, Lecture Notes in Physics 369 (1990).

[15] C. C. Cheung et al., ApJ 826 (2016) 142.

[16] C. C. Cheung et al., ATel 9594 (2016).

[17] R. Ciardullo et al., ApJ 318 (1987) 520.

[18] R. Ciardullo et al., ApJ 356 (1990) 472.

[19] D. D. Clayton and F. Hoyle, ApJ 187 (1974) 101. 
[20] J. G. Cohen, ApJ 292 (1985) 90.

[21] J. G. Cohen, in: The extragalactic distance scale, ASPC 4 (1988) 114.

[22] A. C. Collazzi et al., AJ 138 (2009) 1846.

[23] C. Curtin et al., ApJ 811 (2015) 34.

[24] M. J. Darnley et al., MNRAS 369 (2006) 257.

[25] M. J. Darnley et al., ApJ 833 (2016) 149.

[26] G. de Vaucouleurs, ApJ 223 (1978) 351.

[27] M. Della Valle, in: The extragalactic distance scale, ASPC 4 (1988) 73.

[28] M. Della Valle et el., A\&A 266 (1992) 232.

[29] M. Della Valle et al., A\&A 287 (1994) 403.

[30] M. Della Valle, in Classical Nova Explosions, AIPC 637 (2002) 443.

[31] M. Della Valle and R. Gilmozzi, Sci 296 (2002) 1275.

[32] M. Della Valle and M. Livio, A\&A 286 (1994) 786.

[33] M. Della Valle and M. Livio, ApJ 452 (1995) 704.

[34] M. Della Valle and M. Livio, ApJ 506 (1998) 818.

[35] R. A. Downes and H. W. Duerbeck, $A J 120$ (2000), 2007.

[36] R. A. Downes et al., JAD 11 (2005) 2

[37] H. W. Duerbeck, SSR 45 (1987) 1.

[38] H. W. Duerbeck, MNRAS 258 (1992) 629.

[39] H. W. Duerbeck, AN 330 (2009) 568.

[40] A. Ederoclite, in: Stella Novae: Past and Future Decades, ASPC 490 (2014) 163.

[41] F. Giovannelli and L. Sabau-Graziati, in Proceedings of The Golden Age of Cataclysmic Variables and Related Objects - III, PoS (Golden2015) 001 (2016).

[42] J. A. Graham, in Changing Trends in Variable Star Research, IAU Coll. 46 (1979) 96.

[43] M. A. Guerrero et al., ApJ 857 (2018) 80.

[44] I. Hachisu and M. Kato, ApJSS 167 (2006) 59.

[45] I. Hachisu and M. Kato, ApJ 709 (2010) 680.

[46] K. Hatano et al., MNRAS 290 (1997) 113.

[47] M. Henze et al., A\&A 563 (2014) A2.

[48] N. Henze et al., $A \& A \mathbf{5 8 2}$ (2015) 8.

[49] M. Hernanz, in Stella Novae: Past and Future Decades, ASPC 490 (2014) 319.

[50] R. Hounsell et al., ApJ 734 (2010) 480.

[51] R. Hounsell et al., ApJ 820 (2016) 104.

[52] E. P. Hubble, $A p J 69$ (1929) 103. 
[53] R. Hudec, in Proceedings of The Golden Age of Cataclysmic Variables and Related Objects - III, PoS (Golden2015) 041 (2016).

[54] K. Imamura and K. Tanabe, PASJ 64 (2012) L9.

[55] K. Itagaki et al., ATel 9848 (2016).

[56] P. Jean et al., ATel 11546 (2018).

[57] M. M. Kasliwal et al., ApJ 735 (2011) 94.

[58] M. Kato and I. Hachisu, ApJ 437 (1994) 803.

[59] J. Krautter et al., ApJ 456 (1996) 788.

[60] K.-L. Li et al., ATel 9736 (2016).

[61] K.-L. Li et al., NatAs 1 (2017) 697.

[62] K.-L. Li et al., ATel 10977 (2017).

[63] W. Liller and B. Mayer, PASP 99 (1987) 600.

[64] M. Livio, ApJ 393 (1992) 516.

[65] P. Martin et al., $A \& A 612$ (2018) A38.

[66] F. Matteucci et al., $A \& A 405$ (2003) 23.

[67] B. D. Metzger et al., MNRAS 450 (2015) 2739.

[68] B. D. Metzger et al., MNRAS 457 (2016) 1786.

[69] B. Miszalski et al., MNRAS 456 (2016) 633.

[70] P. J. Morris et al., MNRAS 495 (2017) 1218.

[71] P. Mroz et al., ApJS 219 (2015) 26.

[72] P. Mroz et al., ApJS 232 (2016) 9.

[73] P. Mroz et al., Nat 537 (2016) 649.

[74] https://asd.gsfc.nasa.gov/Koji.Mukai/novae/novae.html

[75] J. U. Ness, BASI 40 (2012) 353.

[76] T. J. O'Brien et al., Nat 442 (2006) 279.

[77] H. Ögelman et al., A\&A 177 (1987) 110.

[78] M. Orio et al., ApJ 466 (1995) 410.

[79] M. Orio, BASI 40 (2012) 333.

[80] M. Orio, in Proceedings of The Golden Age of Cataclysmic Variables and Related Objects - III, PoS (Golden2015) 064 (2016).

[81] J. P. Osborne eta al., ApJ 727 (2011) 124.

[82] J. P. Osborne, JHEA 7 (2015) 117.

[83] A. Özdönmez et al., MNRAS 461 (2016) 1177.

[84] A. Pagnotta, in 20th European White Dwarf Workshop, ASPC 509 (2017) 535. 
[85] J. Patterson, SASS 33 (2014) 17.

[86] C. H. Payne-Gaposchkin, The Galactic Novae, North-Holland Pub. Co. (1957).

[87] W. Pietsch, AN 331 (2010) 187.

[88] R. Poggiani, AN 327 (2006) 895.

[89] R. Poggiani, NewA 13 (2008) 557.

[90] R. Poggiani, Ap\&SS 315 (2008) 79.

[91] R. Poggiani, NewA 14 (2009) 4.

[92] R. Poggiani, AN 330 (2009) 77.

[93] R. Poggiani, Ap\&SS 323 (2009) 319.

[94] R. Poggiani, NewA 15 (2010) 657.

[95] R. Poggiani, NewA 15 (2010) 170.

[96] R. Poggiani, Ap\&SS 333 (2011) 115.

[97] R. Poggiani, MmSAI 83 (2012) 753.

[98] R. Poggiani, NewA 37 (2015) 9.

[99] R. Poggiani, AcPPP 2 (2015) 234.

[100] R. Poggiani,in: Proceedings of The Golden Age of Cataclysmic Variables and Related Objects IV (GOLDEN 2017), POS (GOLDEN 2017) 049 (2018).

[101] R. Poggiani,in: Proceedings of XII Multifrequency Behaviour of High Energy Cosmic Sources Workshop (MULTIF2017) - Astrophysics of High Energy Cosmic Sources, POS (MULTIF 2017) 053 (2018).

[102] G. Ramsay et al., $A \& A 604$ (2017) A107.

[103] H. Ritter and U. Kolb, A\&A 404 (2003) 301.

[104] http://wwwmpa.mpa-garching.mpg.de/RKcat/

[105] E. L. Robinson, AJ 80 (1975) 515.

[106] L. Rosino, AnAp 27 (1964) 498.

[107] L. Rosino, A\&AS 9 (1973) 347.

[108] L. Rosino, AJ 97 (1989) 83.

[109] D. I. Sahman et al., MNRAS 477 (2018) 4483.

[110] B. E. Schaefer, ApJS 187 (2010) 275.

[111] B. E. Schaefer, MNRAS 481 (2019) 3033.

[112] T. Schmidt, Z. Astrophys. 41 (1957) 182.

[113] G. Schwarz et al., ApJS 197 (2011) 31.

[114] P. Selvell and R. Gilmozzi, A\&A 622 (2019) 186.

[115] A. W. Shafter, ApJ 487 (1997) 226.

[116] A. W. Shafter et al., ApJ 530 (2000) 193S. 
[117] A. W. Shafter, in: Classical Nova Explosions: International Conference on Classical Nova Explosions, AIPC 637 (2002) 462.

[118] A. W. Shafter et al., ApJ 734 (2011) 12.

[119] A. W. Shafter et al., ApJ 752 (2012) 156.

[120] A. W. Shafter, AJ 145 (2013) 117.

[121] A. W. Shafter, in: Stella Novae: Past and Future Decades, ASPC 490 (2014) 77.

[122] A. W. Shafter et al., ApJS 216 (2015) 34.

[123] A. W. Shafter, ApJ 834 (2017) 196.

[124] A. W. Shafter and P. K. Irby, ApJ 563 (2001) 749.

[125] M. M. Shara et al., ApJ 311 (1986) 163.

[126] M. M. Shara et al., Nat 446 (2007) 159.

[127] M.M. Shara et al.,ApJ 758 (2012) 121.

[128] M. M. Shara et al., ApJS 227 (2016) 1.

[129] M. M. Shara et al., Nat 548 (2017) 558.

[130] M. M. Shara et al., ApJ 839 (2017) 109.

[131] M. M. Shara et al., MNRAS 465 (2017) 739.

[132] M. M. Shara et al., MNRAS 474 (2018) 1746.

[133] A. S. Sharov, SvA 16 (1972) 41.

[134] A. S. Sharov, AstL 19 (1993) 147.

[135] J. Sitarek and W. Bednarek, PRD 86 (2012) 063011.

[136] J. J. Sokoloski et al., Nat 442 (2006) 276.

[137] S. Starrfield et al., PASP 128 (2016) 051001.

[138] R. J. Strope et al., AJ 140 (2010) 34.

[139] F. Surina et al., arXiv:1111.5524 (2011).

[140] J. Tanaka et al., PASJ 63 (2011) 911.

[141] C. Tappert et al., MNRAS 423 (2012) 2476.

[142] C. Tappert et al., in: Proceedings of The Golden Age of Cataclysmic Variables and Related Objects III, PoS (Golden2015) 062 (2016).

[143] A. B. Tomaney and A. W. Shafter, ApJS 81 (1992) 683.

[144] S. van den Bergh and C. J. Pritchet, PASP 98 (1986) 110.

[145] S. van den Bergh and P. F. Younger, $A \& A S 70$ (1987) 125.

[146] S. van den Bergh, PASP 103 (1991) 609.

[147] I. Vurm and B. D. Metzger, ApJ 852 (2018) 62.

[148] F. M. Walter et al., PASP 124 (2012) 1057. 
[149] F. M. Walter, in Stella Novae: Past and Future Decades, ASPC 490 (2014) 191.

[150] R. E. Williams et al., ApJ 376 (1991) 721.

[151] R. E. Williams, AJ 104 (1992) 725.

[152] R. E. Williams et al., ApJSS 90 (1994) 297.

[153] R. E. Williams, JAD 9 (2003).

[154] R. E. Williams and A. W. Shafter, ApJ 612 (2004) 867.

[155] R. Williams, AJ 144 (2012) 98.

[156] O. Yaron et al., ApJ 623 (2005) 398.

[157] L. Yungelson et al., ApJ 481 (1997) 127. 